\title{
Association between Intention to Stay on the Job and Job Satisfaction among Japanese Nurses in Small and Medium-sized Private Hospitals
}

\author{
Yasushi Kudo ${ }^{1}$, Toshihiko Satoh ${ }^{1}$, Kaori Hosoi ${ }^{1}$, Takeo Miki ${ }^{1}$, Mitsuyasu Watanabe ${ }^{1}$, \\ Shigeri $\mathrm{KIDO}^{2}$ and Yoshiharu AizawA ${ }^{1}$
}

${ }^{1}$ Department of Preventive Medicine and Public Health, Kitasato University School of Medicine and ${ }^{2}$ Department of Fundamentals of Nursing, Kitasato University School of Nursing, Japan

\begin{abstract}
Association between Intention to Stay on the Job and Job Satisfaction among Japanese Nurses in Small and Medium-sized Private Hospitals: Yasushi Kudo, et al. Department of Preventive Medicine and Public Health, Kitasato University School of Medicine-In order to examine the relationship between the intention to stay on the job and job satisfaction among Japanese nurses, and to obtain clues for preventing turnover, we conducted a questionnaire survey. The subjects involved in the survey included 625 female nurses (registered nurses, licensed practical nurses and assistant nurses) working in 4 small and medium-sized private hospitals, excluding directors of nursing. Of the 625 questionnaires distributed, $556(89.0 \%)$ were returned. After excluding the questionnaires with missing values, 480 questionnaires were analyzed (effective response rate, $76.8 \%$ ). The average age of the respondents was $32.8 \mathrm{yr}$ (range: 20-65). The content of the questionnaire was nurse attributes, job satisfaction (30 items) and intention to stay on the job. For job satisfaction, factor analysis (principal factor method and promax rotation) was performed, and factors with an eigenvalue of $\geq 1$ were extracted. Six factors were extracted by factor analysis. These factors were interpreted as "Work as specialists" (1st factor), "Relationship with superiors" (2nd factor), "Comfortable life" (3rd factor), "Relationship among nurses" (4th factor), "Communication with physicians" (5th factor) and "Working conditions" (6th factor). The factor scores were calculated and used as a scale for the evaluation of job satisfaction. To investigate the factors associated with intention to stay on the job among nurses, the
\end{abstract}

Received Feb 24, 2006; Accepted Aug 29, 2006

Correspondence to: Y. Kudo, Department of Preventive Medicine and Public Health, Kitasato University School of Medicine, 1-151 Kitasato, Sagamihara-shi, Kanagawa 228-8555, Japan (e-mail: ykudo@med.kitasato-u.ac.jp) standard partial regression coefficient was computed by multiple linear regression analysis, with intention to stay on the job as the dependent variable, and nurse attributes and job satisfaction (factor scores) as independent variables. Various factors including the organizational culture of each hospital may affect the relationship between job satisfaction and the intention to stay on the job. In order to adjust for these factors, differences among hospitals were included in the statistical model as independent variables. The result of the multiple regression analysis suggests that the intention to stay on the job was higher among nurses who were older and more satisfied with work as specialists (1st factor) and working conditions (6th factor).

(J Occup Health 2006; 48: 504-513)

Key words: Nurse, Turnover, Intention to stay, Job satisfaction, Questionnaire

A high turnover rate among nurses may have unfavorable influences on the nursing system and on the improvement of the quality of nurses trained by extensive clinical experience.

Due to the rapid aging of the population in Japan ${ }^{1)}$, demands for nursing in medical institutions may further increase in the future. Ensuring the manpower of nurses may become an important issue in the management of medical institutions.

Therefore, studies on factors affecting nurses' intention to stay on the job (or turnover intention) are important. In countries outside of Japan, there have been many reports researching the issue of nurse turnover ${ }^{2-6}$.

Alexander et al. examined the processes by which individual characteristics, job satisfaction and intention to quit explained the reasons for nurse turnover in a longitudinal study ${ }^{2}$. According to the path model produced by Alexander et al., dissatisfaction with work 
hazards, relationship with coworkers, professional growth opportunities, role clarity, workload and autonomy were factors deciding nurses' intention to quit. In addition, highly educated nurses and male nurses had stronger intentions to quit.

The intention to quit was the factor augmenting the actual turnover. Moreover, dissatisfaction regarding work hazards and the relationship with coworkers directly increased turnover. Turnover was directly associated with factors such as nurses' ages and their academic levels.

The results of a meta-analysis ${ }^{3)}$ and a cross-sectional study ${ }^{4}$ outside Japan show that the following items were factors triggering nurse turnover: job satisfaction ${ }^{3)}$, advancement opportunity ${ }^{3)}$, group cohesion ${ }^{3)}$, autonomy ${ }^{3)}$, pay $^{3)}$, job stress ${ }^{3)}$, material status ${ }^{3)}$, education level ${ }^{3)}$, professional commitment ${ }^{4)}$, supervisor satisfaction ${ }^{4)}$, organization satisfaction ${ }^{4}$ and stress ${ }^{4}$.

Cavanagh and Coffin reported the results of their crosssectional study examining the factors related to nurses' intention to stay ${ }^{5}$. They reported that high levels of job satisfaction, strong family responsibilities and good pay were associated with a greater likelihood to stay in current employment. Yet, the greater the opportunity individuals have to advance within their field, the less likelihood they have of staying in their current post.

In Japan, a number of recent studies on turnover targeting Japanese nurses have been reported ${ }^{7-10)}$. However, the accumulation of such data is still scarce.

In particular, the study of job satisfaction can be the most useful analytical tool for preventing the resignation of nurses ${ }^{11)}$. However, there have been few studies done on the issue of nurse turnover as it pertains to job satisfaction, including our previous study ${ }^{9-10)}$.

Our previous study presented "job satisfaction" and "nurse attributes" as factors associated with turnover intention and evaluated factors associated with the turnover intention ${ }^{9}$.

The number of nurses with turnover intention was significantly higher among those with low satisfaction regarding sleep, low satisfaction with salary, low satisfaction with welfare, poor implementation of fair salary rises and poor cooperation among nurses. The number of nurses with turnover intention was significantly higher in younger nurses and registered nurses.

In our previous study9), there were only 14 questionnaire items concerning job satisfaction and factor analysis of job satisfaction was not performed.

In this study, we increased the number of the questionnaire items concerning job satisfaction and performed factor analysis of nurses' job satisfaction. We evaluated the association between latent factors concerning job satisfaction and intention to stay on the job among nurses.

The purpose of this study was to increase intention to stay on the job among nurses and obtain clues for preventing turnover. This is the first paper targeting Japanese nurses to discuss the association between the latent factors of job satisfaction and the intention to stay on the job.

Japan has a total of 9,077 hospitals ${ }^{12)}$. Among them, 1,255 hospitals (13.8\%) have less than 50 beds, 2,361 hospitals $(26.0 \%)$ have 50-99 beds, 1,460 hospitals (16.1\%) have $100-149$ beds, 1,244 hospitals (13.7\%) have 150-199 beds, 1,151 hospitals (12.7\%) have 200-299 beds, 775 hospitals (8.5\%) have 300-399 beds, 350 hospitals (3.9\%) have 400-499 beds, and 481 hospitals $(5.3 \%)$ have $500-900$ beds.

One thousand six hundred eighty one hospitals $(18.5 \%)$ are publicly-managed, while 7,396 hospitals (81.5\%) are privately-owned ${ }^{12)}$. In addition, most of the public hospitals are rather large in scale and are responsible for specific duties such as public medical treatment ${ }^{12,13)}$.

In Japan, small and medium-sized private hospitals play central roles in the Japanese medical services industry ${ }^{12,13)}$. In order to fully understand the work environment of Japanese nurses, it is essential to collect data for small and medium-sized private hospitals. This paper studied 4 small and medium-sized private hospitals that had 182299 beds. Hospitals of such a scale comprise about one quarter of the hospitals in Japan.

\section{Methods}

Subjects

The subjects involved in the survey included 625 female nurses (registered nurses, licensed practical nurses and assistant nurses) working in 4 small and mediumsized private hospitals, excluding directors of nursing.

Each hospital was composed of several internal medicine and surgery sections. In each hospital, medical doctors versed in emergency medical care engaged in medical treatment for injured and ill people needing emergency medical care, $24 \mathrm{~h}$ per day, under the direction of prefectural governors. Hospital A had status by the Social Welfare Law of Japan. Hospitals B, C, and D had corporate status by the Medical Care Law of Japan.

In Japan, hospital beds are classified into 5 types, and the statistics regarding hospital beds are recorded ${ }^{12)}$. The 5 classifications are Beds for Psychiatric Patients (beds for patients suffering from psychiatric diseases), Beds for Infected Patients (beds for patients infected with serious infections such as plague and cholera), Beds for Tubercular Patients (beds for tubercular patients), Convalescent Beds (beds for those who need a long convalescence), and General Beds (beds used for purposes different from those mentioned above).

In Japan, there are a total of 1,631,553 hospital beds ${ }^{12}$. In detail, the number of Beds for Psychiatric Patients is 354,927 (21.8\%), the number of Beds for Infected Patients is $1,690(0.1 \%)$, the number of Beds for Tubercular 
Table 1. Characteristics of participating facilities

\begin{tabular}{|c|c|c|c|c|c|c|}
\hline \multirow[b]{3}{*}{ A hospital } & \multirow{3}{*}{$\begin{array}{l}\begin{array}{l}\text { Type of } \\
\text { hospital }\end{array} \\
\text { Private }\end{array}$} & \multirow{3}{*}{$\begin{array}{c}\begin{array}{c}\text { Number of } \\
\text { beds }\end{array} \\
299\end{array}$} & \multicolumn{4}{|c|}{ Number of nurses } \\
\hline & & & Registered nurses & Licensed practical nurses & Assistant nurses & Total \\
\hline & & & $140 \quad(75.3 \%)$ & $(5.4 \%)$ & $36 \quad(19.4 \%)$ & $186(100 \%)$ \\
\hline B hospital & Private & 260 & $162(91.0 \%)$ & $(4.5 \%)$ & $(4.5 \%)$ & $178 \quad(100 \%)$ \\
\hline C hospital & Private & 224 & $90 \quad(62.9 \%)$ & $16 \quad(11.2 \%)$ & $37 \quad(25.9 \%)$ & $143(100 \%)$ \\
\hline \multirow[t]{2}{*}{ D hospital } & Private & 182 & $86 \quad(72.9 \%)$ & $14 \quad(11.9 \%)$ & $18 \quad(15.3 \%)$ & $118 \quad(100 \%)$ \\
\hline & & & Response Rate & Analyzed rate & $\begin{array}{l}\text { Average age } \\
\text { (range) }\end{array}$ & $\begin{array}{c}\text { Number of } \\
\text { married nurses }\end{array}$ \\
\hline A hospital & & & $170 \quad(91.4 \%)$ & $143 \quad(76.9 \%)$ & $36.3(20-60)$ & 84 \\
\hline B hospital & & & $168 \quad(94.4 \%)$ & $150 \quad(84.3 \%)$ & $28.9(20-65)$ & 40 \\
\hline C hospital & & & $112(78.3 \%)$ & $89 \quad(62.2 \%)$ & $33.0(21-58)$ & 29 \\
\hline D hospital & & & $106 \quad(89.8 \%)$ & $(83.1 \%)$ & $33.7(21-65)$ & 41 \\
\hline
\end{tabular}

Table 2. Characteristic of analysis subjects

\begin{tabular}{llrr}
\hline & & $\mathrm{n}$ & $\%$ \\
\hline \multirow{2}{*}{ Age } & $20-29$ & 233 & 48.5 \\
& $30-39$ & 129 & 26.9 \\
& $40-49$ & 73 & 15.2 \\
& $50-$ & 45 & 9.4 \\
\hline \multirow{2}{*}{ Job rank } & Registered nurses & 387 & 80.6 \\
& Licensed practical nurses & 36 & 7.5 \\
& Assistant nurses & 57 & 11.9 \\
\hline \multirow{2}{*}{ With /without night work } & With night work & 323 & 67.3 \\
& Without night work & 157 & 32.7 \\
\hline \multirow{2}{*}{ With /without a spouse } & With a spouse & 194 & 40.4 \\
& Without a spouse & 286 & 59.6 \\
\hline \multirow{2}{*}{ With /without children } & With children & 188 & 39.2 \\
& Without children & 292 & 60.8 \\
\hline \multirow{2}{*}{ Employment status } & Full-time workers & 420 & 87.5 \\
& Part-time workers & 60 & 12.5 \\
\hline Intention to stay on the job & Considerable & 59 & 12.3 \\
& Some & 118 & 24.6 \\
& Intermediate & 141 & 29.4 \\
& Slight & 80 & 17.1 \\
& Negligible & 16.7 \\
\hline \multirow{2}{*}{ Total } & & & 100.0 \\
\hline
\end{tabular}

Patients is $13,293(0.8 \%)$, the number of Convalescent Beds is 349,450 (21.4\%), and the number of General Beds is $912,193(55.9 \%)$. The bed types of each hospital were all General Beds.

In November and December 2005, the nursing director of each hospital distributed anonymous self-administered questionnaires, envelopes for the return of the questionnaires and documents, explaining the purpose of this study, the privacy policy and requested informed consent. About 1 wk after distribution, the completed questionnaires were collected in sealed envelopes at each station, such as wards and clinical departments, by the persons in charge of these stations.

Of the 625 questionnaires distributed, 556 (89.0\%) were returned. After excluding the questionnaires with missing values, 480 questionnaires were analyzed (effective response rate, $76.8 \%$ ). The average age of the respondents was $32.8 \mathrm{yr}$ (range: 20-65) (Table 1 and 2). 


\section{Questions and data analysis}

1) Intention to stay on the job

The question about intention to stay on the job was: "What degree of intention to stay on the job in this hospital do you have?" The subjects selected a response from among: "considerable", "some", "intermediate", "slight", and "negligible." For the statistical analyses, the answers from "considerable" to "negligible" were given 5 to 1 points, respectively.

2) Dimensions of job satisfaction scale items

We prepared 30 original questions regarding job satisfaction with reference to the opinions of the nursing director of each medical institution and from previous studies $^{2-7,9,10,14-25)}$.

The questions about job satisfaction are classified into the following 7 categories: factors related to treatment by the respective medical organization, factors related to comfort, factors related to relationship among nurses, factors related to communication with physicians, factors related to professional commitment, factors related to nursing practice and factors related to self-actualization (Appendix).

For each of the 30 questions, the subjects selected a response from among: "definitely agree", "somewhat agree", "intermediate", "somewhat disagree" and "definitely disagree". For the statistical analyses, the answers from "definitely agree" to "definitely disagree" were given 5 to 1 points, respectively.

Factor analysis (principal factor method and promax rotation) of the 30 question items concerning job satisfaction was performed, and factors with an eigenvalue of $\geq 1$ were extracted. Subsequently, items with a factor loading of $<0.4$ were deleted, and factor analysis (principal factor method and promax rotation) was performed again.

Subsequently, for each item with a factor loading of $\geq 0.4$, explanation was performed. The factor scores were calculated and used as a scale for the evaluation of job satisfaction.

\section{3) Nurse attributes}

The factors related to the basic attributes are as follows: age, job rank (registered nurse, licensed practical nurse and assistant nurse), with / without night work, with / without a spouse, with / without children and employment status (full-time worker or part-time worker).

For statistical analyses, actual age was used. Regarding job ranks, assistant nurses were used as the standard, and dummy variables representing registered nurses and licensed practical nurses were produced. One point was given to full-time workers and subjects with night work, with a spouse and with children. Zero points were given to part-time workers and subjects without night work, without a spouse and without children.

\section{4) Statistical model}

To investigate the factors associated with intention to stay on the job, the standard partial regression coefficient was computed by multiple linear regression analysis, with intention to stay on the job as the dependent variable, and nurse attributes and job satisfaction (factor scores) as independent variables.

Various factors including the organizational culture of each hospital may affect the relationship between job satisfaction and the intention to stay on the job. In order to adjust for these factors, differences among hospitals were included in the statistical model as independent variables.

As we conducted statistical analyses, D hospital, whose bed number was the smallest, was used as the standard and dummy variables representing $\mathrm{A}, \mathrm{B}$, and $\mathrm{C}$ hospitals were produced.

For all analyses, SPSS 11.5 J for Windows was used.

\section{Results}

Table 3 shows the six factors extracted by factor analysis from the 30 items concerning job satisfaction.

The first factor showed loadings of $\geq 0.4$ on: 27 ) Pride in job; 30) Job that can be proudly talked about to others; 28) Personal growth with job; 17) Challenging job; 29) Appreciation received from patients and families; 15) Job having variety; 16) Job with responsibility; and 19) Improvement in ability as specialists.

The second factor showed loadings of $\geq 0.4$ on: 23 ) Superiors' impartialness to nurses; 22) Open communication with superiors; 21) Superiors' appropriate instructions; and 26) Superiors' attitude toward listening to nurses' comments.

The third factor showed loadings of $\geq 0.4$ on: 7 ) Free time; 6) Adequate rest; and 8) Sleep.

The fourth factor showed loadings of $\geq 0.4$ on: 10 ) Nurses on good terms; 11) Mutual help among nurses; and 9) Nurses' teamwork.

The fifth factor showed loadings of $\geq 0.4$ on: 14 ) Physician's cooperation with nurses; 12) Appropriate instructions by physicians; and 13) Open communication with physicians.

The sixth factor showed loadings of $\geq 0.4$ on: 5) Fair salary raise; 1) Salary; and 2) Welfare.

These factors were interpreted as "Work as specialists" (1st factor), "Relationship with superiors" (2nd factor), "Comfortable life" (3rd factor), "Relationship among nurses" (4th factor), "Communication with physicians" (5th factor) and "Working conditions" (6th factor).

Table 4 shows the Pearson's correlation matrix between variables. Intention to stay on the job was significantly correlated with all the variables, excluding the employment status. In addition, significant correlations (0.184-0.609) were observed among the factor scores.

The result of the multiple regression analysis is shown 
Table 3. Promax-rotated principal factor loadings of the job satisfaction

\begin{tabular}{|c|c|c|c|c|c|c|c|c|}
\hline \multirow[t]{2}{*}{ Questions } & \multirow[b]{2}{*}{ Factor 1} & \multirow[b]{2}{*}{ Factor 2} & \multirow[b]{2}{*}{ Factor 3} & \multirow[b]{2}{*}{ Factor 4} & \multirow[b]{2}{*}{ Factor 5} & \multirow[b]{2}{*}{ Factor 6} & \multirow[b]{2}{*}{ Eigenvalue } & \multirow{2}{*}{$\begin{array}{l}\text { Variance } \\
\text { explained }\end{array}$} \\
\hline & & & & & & & & \\
\hline \multicolumn{9}{|l|}{ Factor 1 (Work as specialists) } \\
\hline 27. Pride in job & 0.905 & -0.066 & -0.035 & -0.023 & -0.096 & 0.038 & & \\
\hline 30. Job that can be proudly talked about to others & 0.795 & 0.005 & 0.022 & -0.064 & -0.021 & -0.105 & & \\
\hline 28. Personal growth with job & 0.730 & 0.133 & 0.033 & 0.064 & -0.123 & -0.002 & & \\
\hline 17. Challenging job & 0.722 & -0.018 & 0.048 & 0.039 & 0.060 & 0.015 & & \\
\hline 29. Appreciation received from patients and families & 0.672 & -0.103 & 0.025 & -0.023 & 0.091 & -0.037 & & \\
\hline 15. Job having variety & 0.582 & -0.018 & -0.032 & -0.023 & 0.083 & 0.085 & & \\
\hline 16. Job with responsibility & 0.511 & 0.083 & -0.039 & 0.071 & 0.061 & -0.169 & & \\
\hline 19. Improvement in ability as specialists & 0.446 & 0.104 & -0.057 & -0.005 & 0.035 & 0.278 & 8.135 & 6.138 \\
\hline \multicolumn{9}{|l|}{ Factor 2 (Relationship with superiors) } \\
\hline 23. Superiors' impartialness to nurses & -0.112 & 0.849 & 0.015 & 0.019 & -0.019 & 0.041 & & \\
\hline 22. Open communication with superiors & 0.063 & 0.820 & 0.020 & -0.093 & 0.071 & -0.178 & & \\
\hline 21. Superiors' appropriate instructions & 0.033 & 0.708 & -0.054 & 0.089 & 0.037 & 0.106 & & \\
\hline $\begin{array}{l}\text { 26. Superiors' attitude toward listening to nurses' } \\
\text { comments }\end{array}$ & 0.046 & 0.680 & 0.020 & 0.017 & -0.040 & 0.146 & 2.171 & 5.273 \\
\hline \multicolumn{9}{|l|}{ Factor 3 (Comfortable life) } \\
\hline 7. Free time & 0.010 & 0.011 & 0.878 & -0.001 & 0.005 & -0.016 & & \\
\hline 6. Adequate rest & -0.016 & 0.014 & 0.853 & -0.013 & 0.016 & 0.055 & & \\
\hline 8. Sleep & 0.003 & -0.010 & 0.766 & 0.018 & -0.020 & 0.013 & 1.872 & 3.561 \\
\hline \multicolumn{9}{|l|}{ Factor 4 (Relationship among nurses) } \\
\hline 10. Nurses on good terms & -0.071 & 0.032 & -0.008 & 0.925 & -0.056 & -0.056 & & \\
\hline 11. Mutual help among nurses & 0.067 & -0.037 & -0.025 & 0.863 & -0.038 & -0.009 & & \\
\hline 9. Nurses' teamwork & 0.020 & -0.009 & 0.053 & 0.707 & 0.106 & 0.013 & 1.636 & 4.733 \\
\hline \multicolumn{9}{|l|}{ Factor 5 (Communication with physicians) } \\
\hline 14. Physician's cooperation with nurses & 0.043 & -0.026 & -0.023 & -0.069 & 0.840 & 0.093 & & \\
\hline 12. Appropriate instructions by physicians & -0.022 & -0.044 & -0.011 & 0.071 & 0.811 & 0.094 & & \\
\hline 13. Open communication with physicians & 0.012 & 0.121 & 0.033 & -0.024 & 0.780 & -0.227 & 1.417 & 4.116 \\
\hline \multicolumn{9}{|l|}{ Factor 6 (Working conditions) } \\
\hline 5. Fair salary raise & -0.134 & 0.032 & -0.030 & -0.005 & 0.043 & 0.788 & & \\
\hline 1. Salary & 0.002 & 0.044 & 0.050 & -0.092 & -0.146 & 0.586 & & \\
\hline 2. Welfare & 0.081 & -0.105 & 0.065 & 0.062 & 0.100 & 0.495 & 1.236 & 3.651 \\
\hline
\end{tabular}

Bold-faced type shows factors loadings over 0.400 .

Interfactor correlations

Factor 1 Factor 2 Factor 3 Factor 4 Factor 5 Factor 6

Factor 1 (Work as specialists)
Factor 2 (Relationship with superiors)
Factor 3 (Comfortable life)
Factor 4 (Relationship among nurses)
Factor 5 (Communication with physicians)
Factor 6 (Working conditions)

1

$0.552 \quad 1$

$\begin{array}{lll}0.370 & 0.329 & 1\end{array}$

$\begin{array}{llll}0.504 & 0.540 & 0.314 & 1\end{array}$

$\begin{array}{lllll}0.465 & 0.408 & 0.253 & 0.443 & 1\end{array}$

$\begin{array}{llllll}0.376 & 0.495 & 0.418 & 0.410 & 0.360 & 1\end{array}$ 
Table 4. Pearson's correlation matrix of intention to stay on the job, job satisfaction and nurse attributes

\begin{tabular}{|c|c|c|c|c|c|c|c|c|}
\hline & 1 & 2 & 3 & 4 & 5 & 6 & 7 & 8 \\
\hline 1. Intention to stay on the job & 1 & & & & & & & \\
\hline 2. Work as specialists & $\left.0.490{ }^{* *}\right)$ & 1 & & & & & & \\
\hline 3. Relationship with superiors & $0.362(* *)$ & $0.609\left(^{* *}\right)$ & 1 & & & & & \\
\hline 4. Comfortable life & $0.321(* *)$ & $0.408(* *)$ & $0.370\left(^{* *}\right)$ & 1 & & & & \\
\hline 5. Relationship among nurses & $0.305(* *)$ & $0.557(* *)$ & $0.602\left(^{* *}\right)$ & $0.350\left(^{* *}\right)$ & 1 & & & \\
\hline 6. Communication with physicians & s $0.184(* *)$ & $0.515\left(^{* *}\right)$ & $0.461\left(^{* *}\right)$ & $0.286(* *)$ & $0.494(* *)$ & 1 & & \\
\hline 7. Working conditions & $0.348(* *)$ & $0.444(* *)$ & $0.588\left(^{* *}\right)$ & $0.495\left(^{* *}\right)$ & $0.482\left(^{* *}\right)$ & $0.433\left(^{* *}\right)$ & 1 & \\
\hline 8. Age & $0.407\left({ }^{* *}\right)$ & $0.191(* *)$ & 0.003 & 0.082 & 0.029 & 0.018 & 0.001 & 1 \\
\hline 9. Registered nurses & $-0.274(* *)$ & $-0.160\left({ }^{* *}\right)$ & $\mathrm{r}<0.001$ & $\left.-0.110^{*}\right)$ & -0.041 & -0.077 & -0.017 & $-0.363\left({ }^{* *}\right)$ \\
\hline 10. Licensed practical nurses & $0.154(* *)$ & 0.067 & $-0.112\left(^{*}\right)$ & 0.006 & -0.077 & -0.011 & -0.053 & $0.266(* *)$ \\
\hline 11. With night work & $-0.223\left({ }^{* *}\right)$ & -0.069 & -0.021 & $-.222\left({ }^{* *}\right)$ & -0.011 & -0.055 & -0.045 & $-0.310\left(^{* *}\right)$ \\
\hline 12. With a spouse & $0.275\left({ }^{* *}\right)$ & 0.072 & 0.056 & 0.088 & 0.040 & -0.008 & -0.018 & $0.475(* *)$ \\
\hline 13. With children & $0.294(* *)$ & 0.077 & -0.036 & 0.070 & -0.011 & -0.070 & -0.012 & $0.574\left(^{* *}\right)$ \\
\hline 14. Full-time workers & -0.084 & 0.005 & 0.002 & $-0.145\left(^{* *}\right)$ & 0.018 & 0.049 & 0.009 & $-0.236\left(^{* *}\right)$ \\
\hline 15. A hospital & $0.195(* *)$ & 0.084 & 0.018 & -0.012 & 0.052 & $-0.231\left(^{* *}\right)$ & -0.030 & $\left.0.219^{* *}\right)$ \\
\hline 16. B hospital & $-0.194(* *)$ & 0.089 & 0.044 & -0.077 & 0.037 & $0.374(* *)$ & 0.018 & $-0.256^{(* *)}$ \\
\hline \multirow[t]{2}{*}{ 17. C hospital } & $-0.136\left(^{* *}\right)$ & $-0.197(* *)$ & $-.098\left(^{*}\right)$ & -0.082 & $-0.235\left(^{* *}\right)$ & $-0.197\left({ }^{* *}\right)$ & $-.108\left(^{*}\right)$ & 0.005 \\
\hline & 9 & 10 & 11 & 12 & 13 & 14 & 15 & 16 \\
\hline
\end{tabular}

1. Intention to stay on the job

2. Work as specialists

3. Relationship with superiors

4. Comfortable life

5. Relationship among nurses

6.Communication with physicians

7. Working conditions

8. Age

9. Registered nurses 1

10. Licensed practical nurses $\quad-0.581(* *) \quad 1$

11. With night work $\quad 0.456\left(^{* *}\right) \quad-0.172\left(^{* *}\right) \quad 1$

12. With a spouse $\left.\quad-0.090\left(^{*}\right) \quad 0.072 \quad-0.2311^{* *}\right) \quad 1$

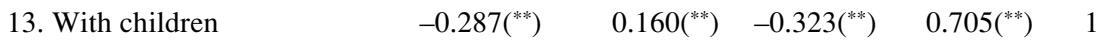

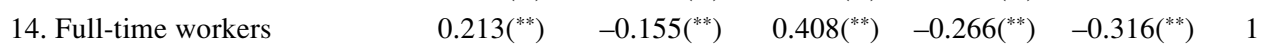

$\begin{array}{llllllll}\text { 15. A hospital } & -0.026 & -0.082 & 0.017 & 0.243\left({ }^{* *}\right) & 0.205\left(^{(* *}\right) & -0.153(* *) & 1\end{array}$

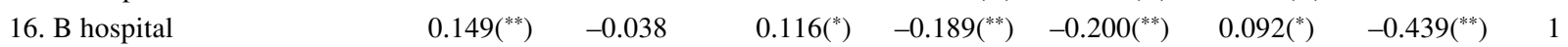

\begin{tabular}{lllllllll} 
17. C hospital & -0.078 & 0.088 & -0.067 & -0.076 & -0.009 & -0.030 & $-0.311(* *)$ & $-0.322(* *)$ \\
\hline
\end{tabular}

${ }^{*}: p<0.05,{ }^{* *}: p<0.01$.

in Table 5. Intention to stay on the job was higher among nurses who were older and more satisfied with work as specialists (1st factor) and working conditions (6th factor). Intention to stay on the job was low among nurses in Hospitals B and C.

\section{Discussion and Conclusion}

Shimizu et al.7) presented "periodical health check-up data", "lifestyle", and "work environment" as factors associated with nurse turnover and evaluated the association between these factors and nurse turnover by longitudinal design. They ${ }^{7)}$ found that sleep disturbance influenced turnover among younger nurses. On the other hand, BMI and T-cho contributed to turnover among older nurses.

Suzuki et al. ${ }^{8)}$ presented "nurse attributes", "burnout", "assertiveness", "stressful life events", "reality shock", "ward assignment preference", "transfer preference", "job satisfaction" (workplace, pay, workload and overtime), "social support" and "coping mechanisms" as factors associated with the turnover of novice nurses and evaluated the association between these factors and 
Table 5. Factors associated with intention to stay on the job among nurses

\begin{tabular}{lcrr}
\hline & Intention to stay & \\
\hline Work as specialists & $0.311^{* * *}$ & VIF \\
Relationship with superiors & 0.104 & $p<0.001$ & 2.230 \\
Comfortable life & 0.044 & 0.052 & 2.341 \\
Relationship among nurses & -0.015 & 0.318 & 1.557 \\
Communication with physicians & -0.081 & 0.755 & 1.963 \\
Working conditions & $0.158^{* *}$ & 0.102 & 2.007 \\
Age & $0.210^{* *}$ & 0.001 & 1.907 \\
Registered nurses & -0.078 & $p<0.001$ & 1.791 \\
Licensed practical nurses & 0.042 & 0.126 & 2.111 \\
With night work & -0.068 & 0.347 & 1.670 \\
With a spouse & 0.083 & 0.125 & 1.611 \\
With children & 0.027 & 0.113 & 2.264 \\
Full-time workers & 0.063 & 0.627 & 2.577 \\
A hospital & -0.006 & 0.118 & 1.344 \\
B hospital & $-0.146^{* *}$ & 0.898 & 1.998 \\
C hospital & $-0.120^{* *}$ & 0.004 & 2.109 \\
\hline Adjusted R square & 0.417 & 0.009 & 1.706 \\
\hline **: $p<0.01$. & & &
\end{tabular}

nurses' turnover by longitudinal design. They ${ }^{8)}$ found that the factors affecting rapid turnover were graduation from vocational nursing schools, dissatisfaction with assignment to a ward contrary to desire and no peers for support.

Shimizu et al..$^{7)}$ and Suzuki et al.$^{8)}$ did not report on the issue of nurse turnover with emphasis on job satisfaction. In contrast, we focused on nurses' job satisfaction, and evaluated the association between latent factors concerning job satisfaction and the intention to stay on the job.

In Japan, there have been some reports on the study of job satisfaction among Japanese nurses ${ }^{14-18}$. However, there have been few reports conducted discussing the issue of nurse turnover with emphasis on nurses' job satisfaction $^{9-10)}$.

$\mathrm{Ozaki}^{21)}$ evaluated job satisfaction among nurses in the U.S. using a job satisfaction questionnaire developed by Stamps et al. ${ }^{22)}$. Since then, many studies have evaluated job satisfaction among Japanese nurses ${ }^{14-17)}$ using the same job satisfaction questionnaire ${ }^{22)}$. This job satisfaction questionnaire ${ }^{22)}$ consists of 7 factors: pay (9 items), professional status (8 items), doctor-nurse relationship (3 items), administration (10 items), autonomy (5 items), task requirement (6 items) and interaction ( 7 items).

However, career formation and medical culture differ between nurses in Japan and those abroad, which may produce a discrepancy in job values. Therefore, it can be considered necessary to produce an original questionnaire about job satisfaction and accumulate the data of its results. Accordingly, we prepared a job satisfaction questionnaire composed of 30 questions, and conducted factor analysis concerning job satisfaction.

In our previous report ${ }^{9)}$, the questions concerning job satisfaction were classified into the following 4 categories: factors related to treatment by the respective medical organization (4 items), factors related to comfort (2 items), factors related to communication (3 items) and factors related to the specialization of nursing ( 5 items). These categories are very general. For instance, the factors related to communication can be further classified into questions regarding physicians (2 items) and a question about nurses ( 1 item). In addition, the latent factors regarding job satisfaction have not been discussed at all.

We initially classified the factors related to forming job satisfaction into 7 categories with reference to the opinions of the nursing director of each medical institution.

The questions about job satisfaction were classified into the following 7 categories: factors related to treatment by the respective medical organization, factors related to comfort, factors related to relationship among nurses, factors related to communication with physicians, factors related to professional commitment, factors related to nursing practice and factors related to self-actualization.

Next, 30 original questions were produced with 
reference to previous studies ${ }^{2-7,9-10,14-25)}$. After that, the nursing director of each medical institution checked the validity of each question in each category. The number of questions was also discussed, and it was concluded that 30 questions would not be a major imposition on the subjects (Appendix).

The factors related to professional commitment (excluding 18) work requiring expert knowledge and techniques) and the factors related to self-actualization were extracted as identical factors (Table 3 ). Because nurses work for the purpose of protecting human life and health, it was considered that professional commitment and self-actualization could not be separated.

To study job satisfaction, the theory of Herzberg et $a l{ }^{25)}$, which is called the two-factor theory, is a very useful analytical tool, and it is also extremely useful for the study of job satisfaction among nurses ${ }^{11,25)}$.

Herzberg et al. $^{25)}$ pointed out the necessity of separating the factors associated with workers' job satisfaction and those associated with dissatisfaction before discussing them because these factors differ from each other. Intrinsic factors that they named 'motivators' (factors intrinsic to the nature and experience of doing work) were associated with job satisfaction and included the following: achievements, recognition, work itself and responsibility. On the other hand, extrinsic factors which they named 'hygiene' were associated with job dissatisfaction and included the following: company policy, administration, supervision, salary, working conditions and relationships with others.

Suzuki et al. ${ }^{8)}$ listed "job satisfaction" as a factor influencing nurse turnover, and produced 4 questions about the workplace, pay, workload and overtime. The usage of the term "job satisfaction" varies according to researchers. The term "job satisfaction" in this paper implies the hygiene factors and motivational factors proposed by Herzberg et al..$^{25)}$.

Intention to stay on the job was significantly associated with the condition of employment (6th factor). The condition of employment is composed of salary, welfare, and fair salary raise, and it is one of the hygiene factors. These factors are associated with the security and stability of subjects' life. The medical organization's attitude to provide security and stability of life to nurses may be an important factor for reducing turnover. This finding is consistent with our previous report targeting nurses in Japan ${ }^{9}$. Previous studies outside Japan also reported that "pay"3,5) included in the concept of hygiene factors is an element which affects turnover, and they are consistent with the results of this study.

Nurses who were satisfied with their work as specialists (1st factor) had stronger intention to stay on the job. This corresponds to the concept of the motivational factors. Nurses work in an effort to protect human life and health, so they are an occupational group with pride. Therefore, since it can be considered that nurses are an occupational group who strongly desire to employ their professional skills and contribute to society, it can be expected that motivational factors will become an important factor in reducing turnover.

Outside Japan, it has been reported that turnover is induced by dissatisfaction with professional growth opportunities $^{2)}$, advancement opportunity ${ }^{3)}$, autonomy ${ }^{2-3)}$ and professional commitment ${ }^{4)}$, which all correspond to the concept of motivational factors. However, Cavanagh and Coffin found that the greater the opportunity individuals have to advance within their field, the less likelihood they have of staying in their current post ${ }^{5}$. Furthermore, in our previous report, turnover intention was not significantly associated with motivational factors ${ }^{9)}$. It is thus necessary to accumulate further data, in future research.

The $p$ value of the relationship with superiors (2nd factor) was 0.052 , although there was no significant relation to the intention to stay on the job. Fang reported that nurses' dissatisfaction with their supervisors was a factor in inducing the turnover intention ${ }^{4}$. It is necessary to further accumulate data in order to study whether the relationship with superiors is significantly related to the intention to stay on the job.

With advancing age, the number of nurses with the intention to stay on the job significantly increased. Nurses should master new medical techniques and deal with various patients. Older nurses appear to have mastered various medical techniques, and acquired abilities and strength to deal with difficult situations in various clinical settings. Careful instruction to young nurses may be important. This result is consistent with our former report $^{9)}$.

Many nurses in Hospitals B and C had little incentive or motivation to stay at their jobs. Therefore, it is considered that there were some factors influencing the intention to stay on the job, other than job satisfaction and the basic attributes of the subjects. Among these factors, organizational culture was considered.

The nursing directors in Hospitals A and D instructed subordinate nurses to have awareness as members of the medical organization and esteem for the medical organization, as well as to improve their nursing skills. Nurses in Hospitals A and D were expected to consider and fulfill their own roles in their organization.

The nursing directors of Hospitals $\mathrm{B}$ and $\mathrm{C}$ also conducted educational seminars or lessons for improving the skills of subordinate nurses. However, the nurses in Hospitals B and C did not receive any education in having esteem for their organization. As a factor different from the degree of job satisfaction, the lack of a sense of belonging to an organization of nurses in Hospitals B and $\mathrm{C}$ may have weakened their intentions to stay at their jobs.

The awareness of contributions to local community 
may be another factor influencing the intention of some nurses to stay at their jobs. The staff at Hospital D had striven to contribute to the local community since the hospital's inception. The nursing director of Hospital A put considerable effort into contributing to the local community. The nurses of Hospitals A and D were expected to have a profound sense of mission to protect the health and lives of local residents. As a factor different from the degree of job satisfaction, the lack of a sense of belonging to a local community of nurses in Hospitals B and $\mathrm{C}$ may have weakened their intentions to stay at their jobs.

In general, organizational culture can influence peoples' behavior ${ }^{26)}$. Such organizational culture nurtured through education may influence the nurses' intentions to stay at their jobs. We would like to address this factor in the future.

A lack of job satisfaction can become a crucial factor in causing a variety of problems such as depression, burnout syndrome and, in extreme cases, suicide. Hereafter, we will address the relationship between the degree of job satisfaction and such problems.

The method applied in this study was a cross-sectional design. Thus, this study is weak in identifying causal relationship, compared with a cohort study. This is one limitation of this paper.

Furthermore, the sampling method of this study did not employ random sampling, limiting the study subjects. This is a second limitation of the study. In future studies, it will be necessary to collect further data regarding the factors associated with nurses' intention to stay on the job.

Acknowledgments: We express deep gratitude to the staff members of the four medical institutions for their cooperation in this study.

\section{References}

1) Ministry of Health, Labour and Welfare. The Latest Trends of Vital Statistics in Japan/Up to 2004. Tokyo: Health and Welfare Statistics Association, 2006 (in Japanese).

2) Alexander JA, Lichtenstein R, Oh HJ and Ullman E: Causal model of voluntary turnover among nursing personal in long-term psychiatric settings. Res Nurs Health 21, 415-427 (1998)

3) Yin JCT and Yang KPA: Nursing turnover in Taiwan: a meta-analysis of related factors. Int J Nurs Stud 39, 573-581 (2001)

4) Fang Y: Turnover propensity and its causes among Singapore nurse: an empirical study. Int J Human Resource Management 12, 859-871 (2001)

5) Cavanagh $S$ and Coffin D: Staff turnover among hospital nurse. J Adv Nurs 17, 1369-1376 (1992)

6) Lu K-Y, Lin P-L, Wu C-M, Hsieh Y-L and Ychang Y$\mathrm{Y}$ : The relationships among turnover intentions, professional commitment, and job satisfaction of hospital nurses. J Prof Nurs 18, 214-219 (2002)
7) Shimizu T, Eto R, Horiguchi I, Obata Y, Feng Q and Nagata $S$ : Relationship between turnover and health check-up data among Japanese hospital nurse: a threeyear follow-up study. J Occup Health 47, 327-333 (2005)

8) Suzuki E, Itomine I, Kanoya Y, Katsuki T, Horii S and Sato C: Factors affecting rapid turnover of novice nurses in university hospital. J Occup Health 48, 4961 (2006)

9) Kudo Y, Satoh T, Sinji H, Miki T, Watanabe M, Wada K, Hosoi K, Hagita K, Saito Y and Aizawa Y: "Factors associated with turnover intention among nurses in small and medium-sized medical institutions". Environ Health Prev Med 11, 128-135 (2006)

10) Matsushita $Y$ and Shibata K: Factors in early retirement of nurses newly graduated from college: motives of the selection of occupation and related factors half a year after employment. Yamanashi Kenritsu Kangodaigaku Kiyou 6, 65-72 (2004) (in Japanese)

11) Lu H, While AE and Barriball KL: Job satisfaction among nurses: a literature review. Int J Nurs Stud 42, 211-277 (2005)

12) Ministry of Health, Labour and Welfare. Survey of Medical Institutions \& Hospital Report (Vol. 1: National Tables) /2004. Tokyo: Health and Welfare Statistics Association, 2006 (in Japanese).

13) Kakihara H. Management of Medical Institutions. In: Introduction to Medical Economics. Tokyo: NipponHyoron-Sha, 2004:169-194 (in Japanese).

14) Takada K, Kusakari J and Kawaguchi T: A study of job satisfaction of nurses in $\mathrm{S}$ university hospital. Nihon Kangokenkyu Gakkaizasshi 18, 53-61 (1995) (in Japanese)

15) Fukazawa K and Kusakari J: Evaluation of nurses' job satisfaction-present status in Shinsyu University Hospital-. Kangokanri 2, 378-383 (1992) (in Japanese)

16) Sekiya C, Takehana K, Nakazawa M, Kamiyama J and Miyashita M: Job satisfaction in nurses at our hospital. Medical J Nagano Red Cross Hospital 10, 45-49 (1996) (in Japanese)

17) Ozaki F and Tadamasa T: A study on the measurement of nurses' job satisfaction in Japan. Osaka Huritsu Kangotandai Kiyou 10, 17-24 (1988) (in Japanese)

18) Simazu N: The structure of international-marketing of medical institutusion-using an investigation of nurses-. Byouinkanri 31, 239-247 (1994) (in Japanese)

19) Chu CI, Hsu HM, Price JL and Lee JY: Job satisfaction of hospital nurses: an empirical test of a causal model in Taiwan. Int Nurs Rev 50, 176-182 (2003)

20) Blegen M: Nurses' job satisfaction: a meta-analysis of related variables. Nurs Res 42, 36-41(1993)

21) Ozaki F: Study on nurses' job satisfaction—comparison between nurses working in ICU/CCU and those working at general wards of internal medicine and surgery in the U.S.- . Jpn J Nurs Res 20, 54-67 (1987) (in Japanese)

22) Stamps PL, Piedmont EB, Slavtt DB and Hasse AM: Measurement of work satisfaction among health 
professions. Med Care 16, 338-341 (1978)

23) Matsuno K: Study on determinant factors affecting burn out in nurses. Shouwa Igakukai Zasshi 47, 383-391 (1987) (in Japanese)

24) Japanese Nursing Association: The survey and analysis of the resignation of hospital nurses and the ways to encourage nurses to stay in the profession. Jpn Nurs Assoc Res Report 44, 7-22 (1994) (in Japanese)

25) Herzberg F, Mausner B and Snyderman B. The Motivation to Work. New York: Wiley, 1958.

26) Robbins PS. Essential of Organizational Behavior, 8th ed. New Jersey: Prentice-Hall, 2004.

Appendix. Dimensions of job satisfaction scale items Factors related to treatment by the respective medical organization

1.I am satisfied with my present salary.

2. There is a good welfare system in this hospital.

3. The principles and ideas of this hospital are good.

4. This hospital has a hospitable atmosphere.

5. Fair salary raises according to ability are implemented in this hospital.

Factors related to comfort

6. I work in this hospital but am able to get adequate rest to relieve fatigue.

7. I work in this hospital but have free time.

8. I work in this hospital but have adequate sleeping time.

Factors related to relationship among nurses

9. In this hospital, there is generally good teamwork among nurses.

10. Nurses in this hospital are on good terms.

11. Nurses in this hospital help one another.

Factors related to communication with physicians 12. Physicians in this hospital generally give appropriate instructions to nurses.

13. I can generally have open communication with physicians about nursing issues.

14. Physicians in this hospital are generally cooperative with nurses.

Factors related to professional commitment

15. My work has much variety, and I am not tired of it.

16. I am in charge of work requiring responsibility.

17. This work is challenging for me.

18. My work requires professional knowledge and techniques.

19. In this hospital, nursing ability can be enhanced.

Factors related to nursing practice

20. In this hospital, assignment to preferred stations is relatively easily granted.

21. My superiors and administrators generally give appropriate instructions to juniors.

22. I can generally have open communication with superiors and administrators about nursing methods.

23. My superiors and administrators generally treat juniors impartially.

24. My workload is appropriate.

25. Nursing work in this hospital is generally performed efficiently.

26. My superiors and administrators generally listen to nurses' proposals.

Factors related to self-actualization

27. While working, I always think that I perform very important work.

28. I feel I achieve personal growth through working in this hospital.

29. I often feel appreciated by patients and their families while working in this hospital.

30. I can proudly talk about my job to others. 